\title{
Pensamiento Complejo en Estudiantes de Ingeniería de Software y su fortalecimiento desde la Cognición Distribuida
}

\section{Complex Thinking in Software Engineering Students and its strengthening from Distributed Cognition}

\author{
Luis Orlando Albarracín-Zambrano \\ p7001262508@ucvvirtual.edu.pe \\ Universidad César Vallejo, Trujillo, Perú \\ Universidad Regional Autónoma de los Andes, Quevedo,Ecuador \\ https://orcid.org/0000-0002-3164-5229
}

Recepción: 10 de mayo 2021

Revisado: 15 de junio 2021

Aprobación: 15 de agosto 2021

Publicación: 01 de septiembre de 2021 


\title{
RESUMEN
}

La formación en ingeniería de software debe promover el pensamiento complejo y las habilidades superiores, para forjar procesos y soluciones innovadoras en torno a las problemáticas en este campo. De allí que la investigación tuvo el propósito de elaborar una propuesta fundamentada en la cognición distribuida para el fortalecimiento del pensamiento complejo en los estudiantes de la asignatura de Automatización. El estudio propositivo siguió un diseño descriptivo-propositivo, desplegándose desde un enfoque metodológico cualitativo. Los sujetos de investigación fueron 30 estudiantes de ingeniería de software de la Universidad UNIANDES-Quevedo, a los que se le aplicó un cuestionario de preguntas abiertas. La cognición distribuida coadyuva a aprender a pensar complejamente, ya que, posibilita una práctica educativa innovadora con cualidad de ser interactiva y de generar aprendizajes de manera colectiva, colaborativa y con la mediación de las TIC y el resto de elementos por donde se distribuye el pensamiento y la cognición.

Descriptores: Pensamiento Complejo; cognición; automatización; Ingeniería. (Palabras tomadas del Tesauro UNESCO).

\begin{abstract}
Training in software engineering must promote complex thinking and superior skills, to forge innovative processes and solutions around the problems in this field. Hence, the research had the purpose of developing a proposal based on distributed cognition for the strengthening of complex thinking in students of the Automation subject. The propositional study followed a descriptive-propositional design, unfolding from a qualitative methodological approach. The research subjects were 30 software engineering students from UNIANDES-Quevedo University, to whom an open-ended questionnaire was applied. Distributed cognition helps to learn to think complexly, since it enables an innovative educational practice with the quality of being interactive and of generating learning in a collective, collaborative way and with the mediation of ICT and the rest of the elements through which thought is distributed and cognition.
\end{abstract}

Descriptors: Complex Thinking, cognition; automation; engineering. (Palabras tomadas del Tesauro UNESCO). 
EPISTEME KOINONIA

Revista Electrónica de Ciencias de la Educación, Humanidades, Artes y Bellas Artes

Año IV. Vol IV. N 8 . Julio - Diciembre. 2021

Hecho el depósito de Ley: FA2018000022

ISSN: 2665-0282

FUNDACIÓN KOINONIA (F.K)

Santa Ana de Coro, Venezuela

Luis Orlando Albarracín-Zambrano

\section{INTRODUCCIÓN}

Actualmente en la educación universitaria y sobre todo en el ámbito de la formación en ingeniería, el proceso de enseñanza aprendizaje es cada vez más susceptible a los cambios, dada las demandas y exigencias del entorno. Por consiguiente, y como expresa Pietro (2007), es menester desarrollar procesos de mejora continua del docente a fin de asegurar la Calidad Educativa en este contexto, lo cual implica ejecutar cambios en los modelos de paradigmas del docente, entendiendo que ahora su tarea principal es la de ser facilitador y mediador en el proceso de construcción de conocimiento de los estudiantes a través de prácticas educativas dinámicas y transformadoras.

Cabe puntualizar que los programas de formación universitaria en ingeniería deben promover el desarrollo del pensamiento y las habilidades de orden superior, a propósito de generar profesionales con capacidad para construir conocimientos y forjar procesos y soluciones innovadoras en torno a las problemáticas en este campo. Esto, devela que el verdadero reto de las carreras de ingenierías consiste en innovar en sus procesos formativos a razón de una "coevolución entre la práctica educativa, el entorno externo, la formación de profesores y la revolución de los planes de estudios" (Serna y Serna, 2017; p. 53).

En este contexto y considerando lo señalado por Mejías et al. (2019), en relación a que las diferentes tecnologías de automatización empleadas en los procesos industriales, permiten contar con sistemas efectivos, robustos y autónomos para la ejecución de dichos procesos. Entonces, se tiene que los programas de formación en ingeniería de software requieren de una nueva mirada en función de generar un profesional capaz de producir este tipo de tecnologías de avanzada para los distintos y cambiantes ámbitos socio humanos. Concretamente, estos programas formativos deben apuntar a que los estudiantes se apropien de aprendizajes y conocimientos solidos sobre automatización y el desarrollo de programas informáticos asociados a la utilización de nuevas tecnologías, plataformas o dispositivos electrónicos para la gestión de diferentes procesos tecnológicos en el mundo industrializado. 
De allí que, en la carrera de ingeniería de software la Universidad Regional Autónoma de Los Andes en su extensión de Quevedo (UNIANDES-Quevedo), se han venido desarrollando diferentes proyectos formativos para la construcción de conocimientos en torno a la automatización en dispositivos que solucionan problemáticas específicas. No obstante, pese al gran esfuerzo de la organización universitaria en este tenor, es necesario el fortalecimiento de las competencias, habilidades y destrezas que tienen los estudiantes, lo que hace evidente que el proceso formativo debe renovarse constantemente, a fin de forjar procesos formativos de calidad y, con ello, generar ingenieros en software con un pensamiento creativo, innovador y crítico.

De acuerdo a lo antes descrito, resulta muy pertinente potenciar las habilidades cognitivas y la forma de pensar de manera compleja en los estudiantes de la carrera de Ingeniería de Software, mediante estrategias didácticas enmarcadas en el modelo o enfoque de la cognición distribuida; aunque, muchos de los programas de formación ingeniería respondan a una orientación muy tradicionalista, la cual, como expresa Estrada (2018), forma a estudiantes con conocimientos limitados, simples y conceptualmente unidisciplinarios, además de llevarlos solo a repetir el conocimiento aprendido sin cuestionar. Esto en palabras de Serna y Serna (2017), significa que "produce en los estudiantes un tipo de pensamiento en el que el conocimiento es fragmentado, compartimentado, mono disciplinar y cuantificado, que conduce a una inteligencia ciega en la medida en que la capacidad humana para conectarlo se sacrifica" (p. 52).

Ahora bien, resulta importante cuestionar ¿qué significa pensar de manera compleja, sobre todo para los estudiantes de ingeniería de software? Pensar complejamente implica asumir que toda realidad como objeto de conocimiento, sea en este campo de la ingeniería o en cualquier otro ámbito del conocer, solo se puede estudiar en relación con su entorno, para lo que es menester superar esquemas de pensamientos que la aíslan, abstraen, reducen y la describen linealmente como una serie de causa-efecto (Morín, 1999). 
De allí que, los estudiantes de ingeniería de software deben apropiarse de la multidimensionalidad y la transdisciplina como modelos de investigación y herramientas fundamentales para pensar complejamente los sistemas. Puesto que, como expresa Serna (2016), la multidimensionalidad permite una comprensión de la realidad como totalidad sistémica constituida por un entretejido de dimensiones inseparables. Así como, la transdisciplina apunta a que todos los conocimientos, habilidades y destrezas de los ingenieros, se consideren herramientas para observar y resolver los problemas del sistema y su entorno de manera más global, para lo que se requiere mayor cooperación, comunicación e integración entre distintas disciplinas, compartiendo mutuamente métodos y temas para gestionar el conocimiento y llegar a una comprensión más profunda de la realidad, desde un pensar de manera colectiva y colaborativa entre los actores.

Cabe destacar que el pensamiento complejo, como expresa Estrada (2018), se forja desde la articulación, la integración de contenidos y la reflexión interior en torno a lo que se piensa y la forma cómo se piensa, por tanto, el aprendizaje se torna reflexivo y sistémico, constituyéndose esto en mecanismo fundamental para que el estudiante despliegue sus capacidades creadoras de conocimientos. Entonces, materializar este tipo de pensamiento demanda estrategias que permitan desarrollar una comprensión profunda de la realidad, pensándola críticamente e integrando conocimiento-acciónreflexión, tal como expresan Pabón y Trigos (2011), desde lo inscrito por Verdejo y Freixas (2009).

Una vía para ello es el desarrollo de tareas auténticas de aprendizaje que representen la complejidad de la realidad, es decir, la realización de quehaceres y actividades, en escenarios reales y/o simulados, pero basados en los problemas prácticos de la vida cotidiana y actuando de manera reflexiva y análoga a los principios epistémicos, pedagógicos y axiológicos de la formación. Esto en virtud de lograr la capacidad de integrar conocimientos, actitudes, habilidades y valores que le permitan abordar como profesional nuevas situaciones problemáticas en escenarios de mayor complejidad e 
incertidumbre (Pabón y Trigos, 2011). Considerando a estos mismos autores, algunas de las estrategias concretas para desarrollar aprendizajes basados en el pensamiento complejo que se pueden listar son:

1. El aprendizaje basado en problemas. Es una metodología que busca desarrollar aprendizajes desde la comprensión de problemas específicos de un contexto particular en el área de estudio; pues, están entrelazados directamente con la práctica profesional o con desempeños futuros del estudiante. Asimismo, el problema es el elemento mediador para el desarrollo de conocimientos y la apropiación de habilidades necesarias para el análisis y la resolución de problemas (Barrows, 1996 en Pabón y Trigos, 2011).

2. El aprendizaje basado en casos contextualizados. Siendo una forma particular de aprendizaje basado en problemas, esta estrategia se apropia de su metodología de abordaje y resolución. Sin embargo, el caso debe narrar una historia real con temas controversiales o dilemas altamente motivadores para los estudiantes (Edelson, 1996 en Pabón y Trigos, 2011).

3. El aprendizaje basado en la reflexión experiencial. Esta estrategia pretende que el estudiante se apropie de algo que es significativo personalmente, al fundamentarse en las experiencias vividas. Por consiguiente, pueden comprender eventos pasados del estudiante, o eventos actuales en los que se ve envuelto con su proceso de aprendizaje. Una experiencia concreta se constituye en ámbito de observación reflexiva, la cual se transforma en comprensión profunda, pues, la dinámica de la reflexión requiere la intervención del intelecto, los sentidos y las emociones, es decir, de la participación del estudiante como persona en su totalidad (Kolb \& Kolb, 2008 en Pabón y Trigos, 2011).

\section{El aprendizaje basado en las Tecnologías de la Información y la} Comunicación (TIC). Esta estrategia conlleva al desarrollo de una educación de tercera generación, con innovadores métodos y técnicas que se producen al estar mediada por las TIC, las cuales ofrecen un amplio espectro de herramientas y 
recursos que facilitan el aprendizaje de conceptos complejos de manera significativa y la construcción de conocimientos, en ambientes interactivos, dinámicos y altamente llamativos (Ordoñez et al., 2011).

5. El aprendizaje basado en la interdisciplinariedad. Esta estrategia se cimienta en la idea de que la construcción del conocimiento por parte de los estudiantes, es mediada por la interdisciplinariedad; por consiguiente, estos son expuestos a abordar y comprender situaciones problemáticas reales, para lo cual se debe asumir los aportes de distintas disciplinas, a fin de confrontar puntos de vista y conocimientos para llegar a soluciones que superen visiones unidisciplinares (Pabón y Trigos, 2011).

6. El aprendizaje colaborativo. Considerando a Carpendale (2009), referenciado por Pabón y Trigos (2011), esta estrategia se funda en la premisa de que el aprendizaje se facilita más en situaciones de interacción donde se coopera para una comprensión mutua entre estudiantes que comparten sus enfoque y perspectivas, los cuales son cuestionados, confirmados o repensados por los miembros del grupo. El aprendizaje colaborativo asiste el desarrollo de habilidades que van más allá de campo disciplinar en estudio, pues, también interviene en los ámbitos: ético, comunicativo, emocional y actitudinal (Ordoñez et al., 2011)

7. El aprendizaje basado en proyectos de investigación. Esta estrategia implica pensar formas de como el estudiante puede aproximarse al conocimiento. Concretamente desarrollar procesos de investigación formativa y/o guiada, convierte al estudiante en actor del proceso, al pretender que sean capaces de realizar actividades que aporten a la solución de problemas contextuales que se abordan en clase como ejercicios formativos, a la vez que se familiaricen con los procesos de la investigación convirtiéndolo en actor del proceso (Restrepo, 2003 en Pabón y Trigos, 2011). 
Considerando lo expuesto, el pensamiento complejo es un enfoque que determina una nueva forma de aprendizaje y de resolución de problemas tangibles de la ingeniería en el mundo real; pues, desarrollar el pensamiento complejo es una posibilidad para una nueva mentalidad caracterizada por la creatividad, el ingenio y la inventiva en el ingeniero como organizador de la información para representar el mundo; por tanto, es un tipo de pensamiento que debe desarrollarse con el proceso formativo de los estudiantes de ingeniería de software (Serna y Serna, 2017).

A propósito de fortalecer este proceso de pensar complejamente los objetos de conocimiento desde la transdisciplina y la multidimensionalidad, es de saber que el enfoque de la cognición distribuida se constituye en una propuesta teórica que promulga, según Salazar (2009) y Resnick y Collins (1996), que las situaciones cognitivas también adoptan la naturaleza de distribuida; es decir, que los procesos cognitivos, que se activan en el individuo para la apropiación de la realidad, se extienden más allá del ámbito del cerebro y las funciones mentales de dicho individuo; pues, la cognición también implica las interacciones con otros individuos en el entorno y está repartida en las herramientas y los artefactos mediadores que se utilizan.

Antes de penetrar en el tema de la cognición distribuida cabe puntualizar que el termino cognición viene del latín "cognoscere", que significa conocer. En este contexto, cabe destacar lo expresado por González y León (2013), en cuanto a que la cognición, la cual se activa a manera de procesos cognitivos, se constituye en el sistema que, estructural y funcionalmente, dispone el proceso de pensamiento el cual dinamiza la construcción y procesamiento de la información que da paso al conocimiento. En términos más llano, la cognición es la habilidad humana que posibilita que las personas se apropien de la realidad, al poder asimilar y procesar los datos que le llegan por diferentes vías y convertirlos en conocimientos.

Ahora bien, la cognición distribuida como teoría se constituye en uno de "los paradigmas más modernos en el campo de las ciencias cognitivas, conocidos en conjunto como 
'ciencia cognitiva corporeizada', 'cognición corporeizada' o 'cognición 4E' (encarnada, embebida, enactiva y extendida), Gissi (2021, s/p).

Esta teoría fue propuesta por el científico cognitivo y antropólogo cultural Edwin Hutchins en los años 80, "para repensar los dominios de la cognición" (Ferruzca, 2008), al enunciar fundamentalmente que el proceso de conocer subsiste no solo en la mente del que aprende, sino también, en el ordenamiento del entorno al distribuirse física, social y simbólicamente; por consiguiente, la construcción de significado se da con la mente social, incorporándose como un todo entorno-acción-artefacto (Nuez, 2018).

La cognición socialmente distribuida implica que se comparte con otros individuos, de allí lo de mente social. En este sistema social-relacional, Resnick y Collins (1996), sostienen que, como referencian Mead (1934) y Vygotski (1978), son dos los elementos de determinantes de la enseñanza; uno, es el concepto de aprendizaje interactivo que postula que el aprendizaje implica la interiorizar procesos y situaciones que previamente han sido practicados en interacción con otros en el entorno; el otro, implica el aprender a interactuar, pues, la competencia cognitiva no solo se da en función de los que se sabe, sino también en función del cómo se usa el conocimiento en situaciones y actividades compartidas con otros. De allí que el trabajo del docente se signifique en crear situaciones de interacción que favorezca la interiorización de estrategias, modos de razonamiento y esquemas conceptuales, tal como refieren Resnick y Collins (1996), desde lo inscrito por Rogoff (1990).

De igual manera y considerando lo enunciado por (Dominio, Catellaro y Roselli, 2010), en este proceso de la cognición socialmente distribuida en situaciones de aprendizaje complejos, los artefactos y herramientas, entendidos como sistema físico constituido materialmente por elementos como: espacio físico, mobiliario, tecnologías disponibles, herramientas informáticas, entre otros, y los convencionalismos socioculturales, asumidos como sistema de instrumentos simbólicos, se establecen como elementos que amplían y prolongan el pensamiento y la capacidad cognitiva humana. Tanto los artefactos físicos como los instrumentos simbólicos permiten "andamiar el conocimiento" 
(Dominio, Catellaro y Roselli, 2010; p.11), generando que el estudiante imagine soluciones y piense de un modo que sería imposible lograrlo sin su acción mediadora. A los efectos de esto, cabe cuestionar ¿de qué manera se puede materializar el proceso de la cognición distribuida en situaciones de aprendizaje complejos? Responder esta interrogante implica asumir, según lo expresado por Jaime (2004), que la cognición distribuida es el resultado del aprendizaje distribuido y este se basa en la colaboración que se establece entre las personas para compartir conocimientos; es decir, busca la creación y gestión del conocimiento colectivo como estrategia. No obstante, esto a su vez depende de la tecnología para compartir, almacenar, recuperar y ampliar el conocimiento, lo que también permite aumenta la capacidad de resolución de problemas; pues, este enfoque teórico, ha sido impactado y dinamizado, en los últimos tiempos, por el sinnúmero de tecnologías de la información y la comunicación.

Significa entonces que la cognición distribuida, la cual se apropia de la idea de que el conocimiento se encuentra diseminado entre el conjunto de personas implicadas en el desarrollo de un proyecto común, se puede concatenar con las distintas estrategias enunciadas con las que se puede desarrollar el pensamiento complejo. Es decir, este proyecto común puede estar representado por cualquiera de las estrategias como el aprendizaje basado en proyectos de investigación interdisciplinaria, que vinculado al aprendizaje reflexivo, colaborativo y mediado por las TIC, se constituye en una posibilidad concreta para desarrollar aprendizajes complejos desde los fundamentos de la cognición distribuida.

Por lo expuesto, y cuestionando ¿Cómo fortalecer el pensamiento complejo en estudiantes de ingeniería de software considerando los fundamentos de la cognición distribuida como enfoque de aprendizaje?, se desarrolló la presente investigación cuyo propósito estuvo enmarcado en elaborar una propuesta fundamentada en el modelo de cognición distribuida para el fortalecimiento del pensamiento complejo en los estudiantes de la asignatura de Automatización del programa de ingeniería de software de la 
Universidad Regional Autónoma de Los Andes en su extensión de Quevedo (UNIANDESQuevedo).

\section{METODOLOGÍA}

Este punto refiere a la metodología sumida para el desarrollo del proceso investigativo. En este tenor (Palella \& Martins, 2015), explican que la metodología, como teoría de la investigación científica que se reflexiona, se constituye en una guía que facilita las pautas lógicas para proceder en cuanto al desarrollo y la coordinación de operaciones destinadas a la consecución de objetivos investigativos y de conocimiento de manera eficaz.

En este contexto y considerando que el diseño de investigación se constituye en el mapa de acciones particulares para lograr los objetivos y/o propósitos de investigación (Estela, 2020), el estudio se enmarcó en ser una investigación propositiva, en correspondencia con un diseño de tipo descriptivo-propositivo. Como se observa claramente, con este tipo de diseño se buscó formular una propuesta de solución enmarcada en los fundamentos de la cognición distribuida, previa realización de un diagnóstico en torno al pensamiento complejo como hecho o fenómeno de estudio.

Cabe resaltar que, la fase descriptiva, la cual se materializa con el diagnostico, se asumió desde un enfoque metodológico cualitativo, el cual se orientó desde la descripción y comprensión de los sentidos y significados, únicos y particulares, de los sujetos participantes de la praxis investigativa (Estela, 2020).

Con respecto al grupo de sujetos participantes de la praxis investigativa, es de saber que estuvo conformado por 30 estudiantes del programa de ingeniería de software de la Universidad Regional Autónoma de Los Andes en su extensión de Quevedo (UNIANDESQuevedo).

Para consolidar la fase de diagnóstico, considerando que se asumió un enfoque de metodología cualitativa, fue necesario aplicar la técnica de la entrevista mediante un cuestionario de preguntas abiertas a propósito de conocer la realidad circundante en 
relación al proceso de pensar complejamente los objetos de aprendizaje de la asignatura de Automatización del programa de ingeniería de software que, a su vez, sirvió de base para el desarrollo de la propuesta.

\section{RESULTADOS Y DISCUSIÓN}

\section{Fase Descriptiva}

En esta fase de descripción diagnóstica, la cual se desarrolló desde una perspectiva cualitativa, se constituye en el ámbito de desvelamiento e interpretación de la realidad emergente en torno al pensamiento complejo. Por consiguiente, pare ello fue necesario aplicar un cuestionario de preguntas abiertas a los estudiantes de ingeniería de software como forma de indagar su experiencia vivida en el aula en torno a pensar complejamente en los procesos de automatización como objetos de aprendizaje y conocimiento. Pues, ello posibilito el desvelamiento de las sentidos y significados que, a manera de necesidades, requieren ser abordadas desde los siguientes temas categoriales emergentes:

PROCESO DIDÁCTICO CONVENCIONAL. En lo que respecta a los procesos didácticos que desarrollan los docentes, se desvela que estos son percibidos por los estudiantes como muy tradicionalistas, es decir, el modelo didáctico se define como informativo, pues, las actividades de aprendizaje están centrada en la transmisión y reproducción de información, dándole al docente la responsabilidad del proceso y, por lo contrario, poca autonomía $y$ participación del estudiante, limitándolo en la construcción de su propio conocimiento de manera creativa y crítica, tal como enuncia Estrada (2018).

PENSAMIENTO COMPLEJO. Desde la viva voz de los estudiantes, se manifiesta la necesidad de abordar los procesos de automatización y de desarrollo de programas informáticos de manera integrativa, es decir desde la integración $y$ articulación de contenidos y no con una visión disciplinar. Para ello, se requiere de cambio en los modelos de paradigmas educativos que implique la 
superación de procesos didácticos que conllevan a un pensamiento lineal, que reduce y aísla, para asumirlos ahora desde una perspectiva transdisciplinar, pues, esto permite que aflore las capacidades creativas $y$ de innovación en los estudiantes, al momento de observar y pensar dichos procesos como objetos compleios de conocimiento, desde toda su multidimensionalidad que lo constituyen y que, además, induzca al propio estudiante a reflexionar y a hacer crítica de su propia forma de pensar en la experiencia formativa, planteamientos que colindan con lo expresado por Estrada (2018) y Serna (2016).

ESTRATEGIAS INNOVADORAS E INTERACTIVAS. Desde lo expresado por los estudiantes, se puede enunciar que desarrollar procesos de aprendizajes centrados en el pensamiento complejo implica un cambio en la estrategia que se despliega con el acto didáctico. En tal sentido, estás estrategias deben estar fundamentadas en el proceso de interacción, es decir en la interrelación que permita desarrollar aprendizajes entre estudiantes de manera compartida $\boldsymbol{y}$ colaborativa, además, de la interconexión que se logre con el uso de las tecnologías y las herramientas de la información y la comunicación para un aprendizaje mediado. De igual manera, este tipo de aprendizaje complejo se debe desarrollar en escenarios reales y mediante proyectos basados en los problemas de automatización que afectan de la realidad tecnológica, organizacional y humana. Situación tal, se compagina con lo expresado por Pabón y Trigos, (2011).

Para cerrar la fase descriptiva, cabe decir que los resultados que se obtuvieron, descubren la necesidad que existe de realizar cambios en el proceso didáctico si se aspira formar un estudiante que piense complejamente los objetos de aprendizaje y con ello desarrolle procesos creativos e innovadores capaces de extrapolarlos a otros contextos como profesional de la ingeniería de software. Asimismo, estas categorías develadas justifican el desarrollo de estrategias innovadoras, que se pueden estructurar 
desde la cognición distribuida que apunta a considerar como principios fundamentales los procesos de interacción humana con los distintos elementos de la realidad.

\section{Fase Propositiva}

\section{PROPUESTA}

La Cognición Distribuida para el Fortalecimiento del Pensamiento Complejo: Modelo de Capacitación para Estudiantes de Ingeniería de Software

\section{Presentación}

En la actualidad, es menester que los estudiantes de ingeniería de software fortalezcan y potencien las habilidades de pensar complejamente los objetos de aprendizaje desde toda su multidimensionalidad. No obstante, para ello se requiere que los docentes desplieguen estrategias didácticas bien fundamentadas e innovadoras que conlleven a superen la visión unidisciplinar y trascender a lo inter y transdisciplinar; pues, pensar complejamente toda realidad como objeto de conocimiento solo es posible hacerlo desde su interacción e interrelación con el entorno; en este contexto, el modelo o enfoque de la cognición distribuida se constituye en una posibilidad para ello.

De allí que, "La Cognición Distribuida para el Fortalecimiento del Pensamiento Complejo", se presenta como propuesta viable, pues, asume como principio fundamental que los procesos de interacción humana con los distintos elementos de la realidad son indispensables para desarrollar la cognición extendida y el conocimiento compartido.

\section{Objetivo}

Fortalecer las habilidades de pensamiento complejo desde los fundamentos de la cognición distribuida, en los estudiantes de la asignatura de Automatización del programa de ingeniería de software de la Universidad UNIANDES-Quevedo. 


\section{Justificación}

Considerando los resultados del diagnóstico, que responden a la fase descriptiva, se justifica el desarrollo de la propuesta "La Cognición Distribuida para el Fortalecimiento del Pensamiento Complejo", en estudiantes del programa de formación en ingeniería de software de la universidad UNIANDES-Quevedo. Con relación a estos resultados, cabe decir, que estos muestran un proceso didáctico que se despliega en la asignatura de automatización, caracterizado como convencional, tradicionalista, centrado en la transmisión de información y que le otorga poca autonomía y participación al estudiante para que sea responsable de su proceso de autogestión del aprendizaje y conocimientos. Asimismo, para superar esta situación impera la necesidad de fortalecer el pensamiento complejo, mediante estrategias interactivas, integradoras, reflexivas, colaborativas y de creación de conocimientos y aprendizajes compartidos, que lleven a los estudiantes de ingeniería de software a aflorar sus capacidades creativas e innovadoras en torno a producir tecnologías de avanzada en el campo de la automatización y el desarrollo de programas informáticos, asociados a la utilización de nuevas tecnologías, para los distintos ámbitos socio humanos y organizacionales.

\section{Fundamentación Pedagógica}

La propuesta que se esboza para fortalecer el Pensamiento Complejo, en estudiantes del programa de formación en ingeniería de software de la universidad UNIANDES-Quevedo, está fundamentada pedagógicamente desde los principios del enfoque teórico de la cognición distribuida, cuyo máximo exponente es el científico cognitivo y antropólogo cultural Edwin Hutchins.

Cabe destacar que se asume esta perspectiva por ser uno de los paradigmas más innovadores de las ciencias cognitivas, que posibilita la sustentación de una práctica educativa y de aprendizaje vigorizadora del proceso de pensar complejamente los objetos de aprendizaje y conocimiento, en los estudiantes de ingeniería de software. 
En este contexto y considerando que dichos estudiantes deben desarrollar nuevos esquemas de pensamiento complejo, la estrategia de aprendizaje distribuido para pensar complejamente los objetos de la realidad, se ha diseñado fundamentándose en los siguientes principios:

1. La interacción. La interacción es fundamental, pues, el aprendizaje se da con la mente social. Lo que quiere decir, que aprender implica interiorizar procesos y situaciones que antes se han dado en la interacción con otros en el entorno, formal e informal, de aprendizaje.

2. La colaboración. Materializar el aprendizaje interactivo implica asumir una postura colaborativa, ya que, es la forma como los estudiantes pueden distribuir pensamientos y con ello gestionar conocimientos y aprendizajes de manera compartida colectivamente.

3. El proceso de andamiaje. En el proceso de aprender complejamente los objetos de aprendizaje en ingeniería de software son esenciales, tanto los elementos físicos (las tecnologías, herramientas informáticas, los materiales tecnológicos, entre otros), como los instrumentos simbólicos que representan al conocimiento (por ejemplo: los tipos de lenguaje de programación), pues, estos se constituyen en elementos mediadores y plataformas para dicho aprendizaje, dado que, ahí también está distribuido la información y el conocimiento.

4. Las TIC. Estas se constituyen en elementos de andamiaje para el desarrollo de aprendizajes complejos, en el sentido de que amplían y diseminan las informaciones y los conocimientos.

5. El problema. El problema es el punto de partida para la construcción de conocimientos, a la vez que se constituye en elemento mediador que permite la apropiación de habilidades necesarias para el análisis y la resolución de problemas complejos de la realidad tecnológica en las organizaciones socio humanas, como objetos de aprendizaje en este caso. 
6. La multidimensionalidad. Pensar complejamente los problemas de la realidad tecnológica en las organizaciones socio humanas como objetos de aprendizaje implica, esencialmente, asumirlos como un entretejido de dimensiones inseparables, por consiguiente, se han de problematizar y comprender como totalidad.

7. La transdisciplinariedad. Abordar los problemas de la realidad tecnológica desde sus múltiples dimensiones demanda asumir una postura transdisciplinar. Es decir, considerar y hacer apropiación de los aportes de los distintos campos disciplinares y su conexión e integración para lograr esa comprensión compleja multidimensional.

8. La reflexión. Pensar complejamente objetos de aprendizaje, desde una postura transdisciplinar, también envuelve reflexionar lo que es significativo en el sujeto. Es decir, implica hacer observación reflexiva y autocritica de lo que se piensa y cómo se piensa ante los problemas de la realidad. Esto transforma el aprendizaje, la comprensión y, por ende, la evaluación en procesos profundos que integran las distintas dimensiones (intelecto, sentidos, acciones, emociones, relaciones), del ser humano.

9. El proyecto de desarrollo tecnológico. Representa el modelo estructural y organizado de todas las actividades a realizarse con base en los principios asumidos y las necesidades de aprendizaje complejo en el área de automatización de los estudiantes.

10. El docente como estratega. Pues, su rol consiste en crear escenarios y situaciones educativas innovadoras, para abordar problemas complejos como objetos de aprendizaje fundadas en la interacción y en la conexión con elementos mediadores, asumidos como andamios para la distribución del pensamiento y la construcción de conocimientos y aprendizajes.

11. El estudiante como ente activo. Pues, de su capacidad de interactuar colaborativamente con otros en situaciones problemáticas y de conectarse con la 
información que se distribuye a través de los distintos elementos mediadores, depende el cambio de su pensamiento y, con ello, el desarrollo de nuevos conocimientos y aprendizajes.

\section{Ámbito de desarrollo y Modalidad-Duración de la propuesta}

El ámbito de desarrollo de la propuesta "La Cognición Distribuida para el Fortalecimiento del Pensamiento Complejo", es la universidad UNIANDEZ del Ecuador, específicamente en la extensión Quevedo; la cual es una institución de educación universitaria privada fundada en el año 1996 a fin de "formar profesionales de tercer y cuarto nivel, de investigación, responsables, competitivos, con conciencia ética y solidaria, capaces de contribuir al desarrollo nacional e internacional, mediante una educación humanista, cultural y científica dirigida a bachilleres y profesionales nacionales y extranjero" (UNIANDES, 2020).

Cabe resaltar que dicha propuesta está dirigida específicamente a los estudiantes de la asignatura Automatización del programa de formación en ingeniería de software. De igual forma, dicha propuesta está organizada en 10 encuentros de aprendizaje aunado a 5 encuentros de actividades prácticas o talleres, que van de 3 o más horas de trabajo académico, considerando el objetivo y las estrategias adoptadas para cada sesión, las cuales serán mediadas por las TIC, además, de las necesidades que emerjan de los estudiantes participantes del proceso formativo.

\section{Plan de Desarrollo Operativo}

Considerando los principios definidos desde la cognición distribuida para fortalecer el pensamiento complejo en estudiantes de la asignatura Automatización del programa de ingeniería de software, los encuentros de aprendizajes que constituyen el plan operativo, quedaron estructurados de la siguiente manera (ver tabla 1): 


\section{Tabla 1.}

Estructura Organizativa de los encuentros de aprendizajes.

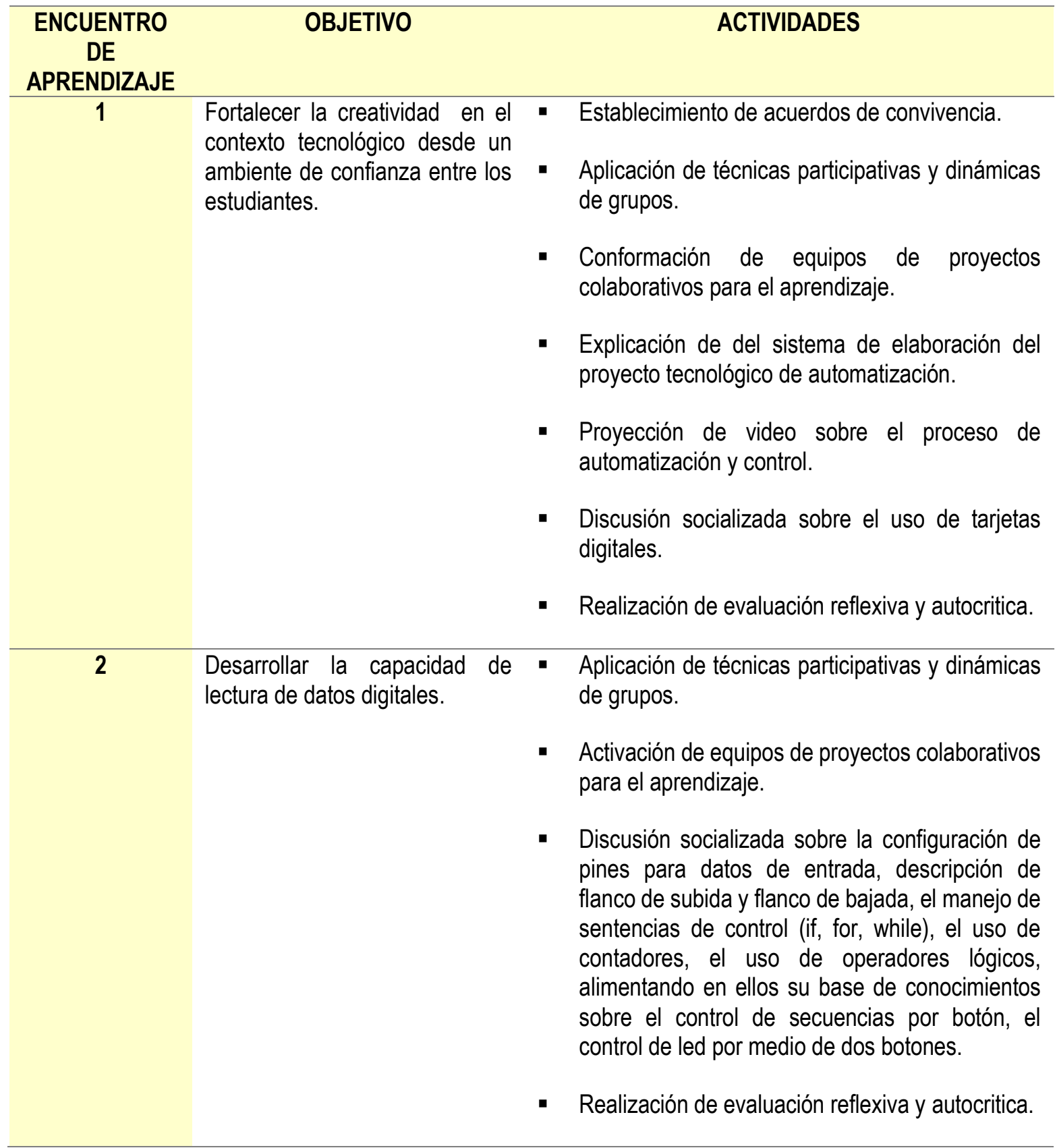


3 Desarrollar la capacidad de lectura de datos analógicos.

Aplicación de técnicas participativas y dinámicas de grupos.

- Activación de equipos de proyectos colaborativos para el aprendizaje.

- Discusión socializada sobre la arquitectura de los pines de lectura analógica dentro de la tarjeta Arduino, la lectura de potenciómetros sensores.

- Realización de evaluación reflexiva y autocritica.

4 Desarrollar la capacidad creativa del estudiante mediante la comprensión de la comunicación serial.
- Aplicación de técnicas participativas y dinámicas de grupos.

- Activación de equipos de proyectos colaborativos para el aprendizaje.

- Discusión socializada sobre la comunicación serial, sus principales características, las funciones usadas por Arduino y el uso de variables que configuran los puertos para comunicación serial y el envío de datos dentro de una tarjeta digital.

- Realización de evaluación reflexiva y autocritica.

5
Fortalecer la capacidad para innovar en la solución de problemáticas automatización.

- Aplicación de técnicas participativas y dinámicas de grupos.

de - Activación de equipos de proyectos colaborativos para el aprendizaje.

- Discusión socializada sobre los problemas de automatización.

- Lluvia de ideas sobre innovaciones, apropiándose de conocimientos sobre el uso de librerías y las características del uso de una pantalla LCD

- Realización de evaluación reflexiva y autocritica.

$6 \quad$ Fortalecer la capacidad para innovar en la solución de problemáticas automatización.

- Aplicación de técnicas participativas y dinámicas de grupos.

de - Activación de equipos de proyectos colaborativos para el aprendizaje.

- Discusión socializada sobre las características del control PWM, el uso de servomotores y sus 


\begin{tabular}{|c|c|c|}
\hline & & $\begin{array}{l}\text { características principales, el control de un motor, } \\
\text { su funcionamiento y su utilidad. } \\
\text { - Realización de evaluación reflexiva y autocritica. }\end{array}$ \\
\hline 7 & $\begin{array}{l}\text { Fortalecer la capacidad para } \\
\text { innovar en la solución de } \\
\text { problemáticas }\end{array}$ & $\begin{array}{l}\text { - Aplicación de técnicas participativas y dinámicas } \\
\text { de grupos. } \\
\text { - Activación de equipos de proyectos colaborativos } \\
\text { para el aprendizaje. } \\
\text { - Discusión socializada sobre las características } \\
\text { del control PWM, el uso de servomotores y sus } \\
\text { características principales, el control de un motor, } \\
\text { su funcionamiento y su utilidad. } \\
\text { - Realización de evaluación reflexiva y autocritica. }\end{array}$ \\
\hline 8 & $\begin{array}{l}\text { Fortalecer la capacidad de } \\
\text { inventiva en los procesos de } \\
\text { automatización. }\end{array}$ & $\begin{array}{l}\text { - Aplicación de técnicas participativas y dinámicas } \\
\text { de grupos. } \\
\text { - Activación de equipos de proyectos colaborativos } \\
\text { para el aprendizaje. } \\
\text { Discusión socializada sobre la aplicación de las } \\
\text { tarjetas digitales Arduino y su uso en la domótica } \\
\text { como solución a procesos de automatización. } \\
\text { - Establecimiento de acuerdos para la realización } \\
\text { talleres prácticos para el desarrollo del proyecto } \\
\text { tecnológico. } \\
\text { - Realización de evaluación reflexiva y autocritica. }\end{array}$ \\
\hline 9 & $\begin{array}{l}\text { Fortalecer la capacidad de } \\
\text { inventiva en los procesos de } \\
\text { automatización }\end{array}$ & $\begin{array}{l}\text { - Aplicación de técnicas participativas y dinámicas } \\
\text { de grupos. } \\
\text { - Activación de equipos de proyectos colaborativos } \\
\text { para el aprendizaje. } \\
\text { - Proyección de video y discusión socializada sobre } \\
\text { las tarjetas raspberry Pi y su sistema operativo. } \\
\text { - Desarrollo de practica sobre instalación de } \\
\text { sistemas operativos, software y drivers para el } \\
\text { manejo de las tarjetas. } \\
\text { - Realización de evaluación reflexiva y autocritica. }\end{array}$ \\
\hline 10 & $\begin{array}{l}\text { Fortalecer la capacidad de } \\
\text { inventiva mediante la generación } \\
\text { de productos tecnológicos }\end{array}$ & $\begin{array}{l}\text { - Aplicación de técnicas participativas y dinámicas } \\
\text { de grupos. }\end{array}$ \\
\hline
\end{tabular}


OBSERVACION: Este encuentro de aprendizaje se diversifica en 5 talleres prácticos.
- Activación de equipos de proyectos colaborativos para el aprendizaje.

- Despliegue de talleres prácticos para la generación de productos tecnológicos.

- Presentación de producto tecnológico

Fuente: Adaptado de Albarracín (2021)

\section{CONSIDERACIONES FINALES}

En la actualidad, los programas de formación universitaria en Ingeniería de Software deben superar la visión educativa de formar a estudiantes desde lo unidisciplinar como tradición enquistada, que limita y cercena el pensamiento, condenando a solo reproducir la información sin cuestionamiento. Por lo contrario, deben apuntar al desarrollo de habilidades cognitivas de orden superior que lleve a los estudiantes a apropiarse las distintas realidades en toda su multidimensionalidad y a pensarlas, como objetos de conocimientos y aprendizajes, de manera compleja y de forma transdisciplinar.

De allí que la investigación estuvo enmarcada en elaborar una propuesta fundamentada en el modelo de cognición distribuida para el fortalecimiento del pensamiento complejo en los estudiantes de la asignatura de Automatización del programa de ingeniería de software de la universidad UNIANDES-Quevedo. Pues, los resultados del diagnóstico descriptivo develan una necesidad de cambio en la estrategia didáctica, la cual se percibe como convencional y muy tradicionalista, en este contexto de aprendizaje. Esto, a fin de que dichos estudiantes sean capaces de pensar los problemas de la realidad contextual de manera compleja y en toda su multidimensionalidad, pudiendo desarrollar procesos creativos e innovadores con su abordaje.

Asimismo, la cognición distribuida se asume como fundamento para desarrollar aprendizajes, pues posibilita una práctica educativa innovadora que se forja en su concreción real desde la: 
- Interacción

- Construcción colectiva y colaborativa

- Mediación de las TIC y el resto de elementos por donde se distribuye el pensamiento y la cognición

- Problematización de las situaciones reales y contextuales

- Multidimensionalidad

- Transdisciplinariedad

- Práctica de la reflexión

- Ejecución de proyecto de desarrollo tecnológico

- Transformación del docente en estratega

- Trascendencia del estudiante como ente activo en la construcción de su propio aprendizaje y conocimiento.

\section{FINANCIAMIENTO}

No monetario.

\section{AGRADECIMIENTO}

A la Universidad Regional Autónoma de los Andes, Quevedo, Ecuador y la Universidad César Vallejo, Trujillo, Perú; por motivar el desarrollo de la investigación.

\section{REFERENCIAS CONSULTADAS}

Albarracín, L. (2021). Programa de aprendizaje fundamentado en la cognición distribuida de Hutchins, para fortalecer el pensamiento complejo de estudiantes en ingeniería de software (Tesis Doctoral) [Hutchins Distributed Cognition-Based Learning Program to Strengthen Complex Thinking of Software Engineering Students]. Recuperado de https://repositorio.ucv.edu.pe/handle/20.500.12692/68863 
EPISTEME KOINONIA

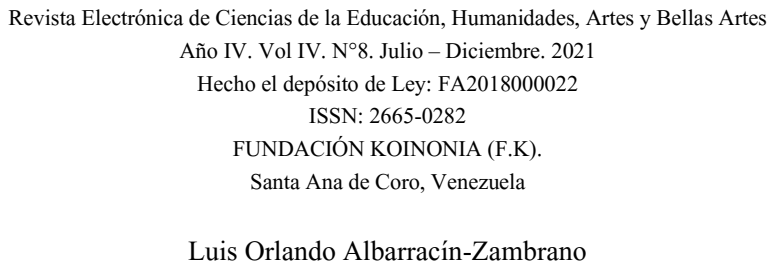

Dominio, M., Catellaro, M. y Roselli, M. (2010). Los sistemas de cognición distribuida en la enseñanza universitaria en función del tipo de ciencia [Distributed cognition systems in university education according to the type of science]. Revista de Psicología UCA, 6(11), 7-39.

Estela, R. (2020). Investigación propositiva [Purposeful research]. Recuperado de https://n9.cl/j3ap

Estrada, A. (2018). Pensamiento complejo y desarrollo de competencias transdisciplinares en la formación profesional [Complex thinking and development of transdisciplinary competences in vocational training]. CIEG, 31, 98-108. Recuperado de https://n9.cl/569bm

Ferruzca, M. (2008). Estudio teórico y evidencia empírica de la aplicación del marco teórico de "Cognición Distribuida" en la gestión de sistemas de formación eLearning. (Tesis Doctoral) [Theoretical study and empirical evidence of the application of the theoretical framework of "Distributed Cognition" in the management of e-Learning training systems.]. Universidad Politécnica de Catalunya, España. Recuperado de https://n9.cl/v0wg0v

Gissi, S. (16 de agosto de 2021). La teoría de la cognición distribuida de Edwin Hutchins y la memoria colectiva. [Edwin Hutchins' theory of distributed cognition and collective memory] [Mensaje en un blog]. Recuperado de https://n9.cl/ucwvv

González, B. y León, A. (2013). Procesos cognitivos de la prescripción curricular a la praxis educativa [Cognitive processes from curricular prescription to educational praxis]. Revista de Teoría y Didáctica de las Ciencias Sociales, 19, (49-67). Recuperado de https://www.redalyc.org/pdf/652/65232225004.pdf

Jaime, M. (2004). Aprendizaje distribuido: una respuesta a las necesidades de la enseñanza de la física en educación secundaria [Distributed learning: a response to the needs of physics teaching in secondary education]. (Tesis de Maestría). Instituto Tecnológico y de Estudios Superiores de Monterrey, México. Recuperado de https://n9.cl/w7zmz

Mejía, Á., Jabba, D., Carrillo, Garyn y Caicedo, J. (2019). Influencia de la Ingeniería de Software en los Procesos de Automatización Industrial [Influence of Software Engineering in Industrial Automation Processes]. Información tecnológica, 30(5), 221-230. DOI https://dx.doi.org/10.4067/S0718-07642019000500221 
Morín, E. (1999). Introducción al pensamiento complejo [Introduction to complex thinking]. Buenos Aires: Gedisa.

Nuez, G. (2018). Acercamiento critico interpretacional al texto: cómo leen los docentes, dinámicas de conceptualización y emergencia de patrones sociales [Critical interpretive approach to the text: how teachers read, conceptualization dynamics and emergence of social patterns]. En B. Cantalapiedra, P. Aguilar y P. Requeijo (Coords.), Formulas docentes de vanguardia (189-204). España. Recuperado de https://n9.cl/6vd7z

Ordoñez, G. et al. (2011). Estrategias para el desarrollo de competencias y pensamiento complejo en el aula en asignaturas del área de ingenierías [Strategies for the development of competences and complex thinking in the classroom in subjects in the engineering area]. En InnovaCesal (Ed.), Estrategias para el desarrollo de pensamiento complejo y competencias: Sistematización de experiencias y buenas prácticas de docentes universitarios (19-66). Veracruz, México. Recuperado de https://n9.cl/by1rb

Palella, S. \& Martins, F. (2015). Metodología de la Investigación Cuantitativa [Quantitative Research Methodology]. Caracas. FEDUPEL

Pabón, N. y Trigos, L. (2011). Estrategias y orientaciones para la formación en competencias y pensamiento complejo [Strategies and guidelines for training in complex thinking and competencies]. En InnovaCesal (Ed.), Estrategias para el desarrollo de pensamiento complejo y competencias: Sistematización de experiencias y buenas prácticas de docentes universitarios (19-66). Veracruz, México. Recuperado de https://n9.cl/by1rb

Prieto, L. (2007). Autoeficacia del profesor universitario: eficacia percibida y práctica docente [Self-efficacy of the university professor: perceived efficacy and teaching practice]. Narcea Ediciones.

Resnick, L. y Collins, A. (1996). Cognición y aprendizaje [Cognition and learning]. Anuario de Psicología, 69 (189-197).

Salazar, P. (2009). Implicaciones de la cognición distribuida y situada en el aprendizaje [Implications of distributed and situated cognition in learning]. (Tesis de Maestría). Universidad de Chile, Chile. Recuperado de https://n9.cl/rlzgy 
Serna, E. (diciembre de 2016). El Pensamiento Complejo para la Ingeniería de Requisitos [Complex Thinking for Requirements Engineering]. EL PENSAMIENTO COMPLEJO: Los desafíos en un mundo globalizado. Congreso mundial de Pensamiento Complejo, Paris, Francia. Recuperado de https://n9.cl/n1sdz

Serna, E. y Serna, A. (2017). Complejidad y Pensamiento Complejo para innovar los procesos formativos en ingeniería [Complexity and Complex Thinking to innovate training processes in engineering]. Sistemas, Cibernética e Informática, 14 (1), 4855. Recuperado de https://n9.cl/fz1p2s

UNIANDES (2020). Plan Estratégico de Desarrollo Institucional 2020-2024 [Strategic Plan for Institutional Development]. Recuperado de https://n9.cl/f6pmb 